\title{
Serum metabolomic investigations of mulberry leaf powder supplementation in Chinese Erhualian pigs
}

\author{
S. Deng ${ }^{1}$, D. Li ${ }^{1}$, X. Liu' ${ }^{1}$, Z. Cai ${ }^{2}$, W. Wei ${ }^{1}$, J. Chen ${ }^{1}$ and L. Zhang ${ }^{1,3}$ \\ ${ }^{1}$ Nanjing Agricultural University, College of Animal Science and Technology, No.1 Weigang, 210095, Nanjing, China \\ ${ }^{2}$ Zhejiang Chinese Medical University, Laboratory Animal Research Center, No.548 Binwen Road, 310053, Hangzhou, China
}

KEY WORDS: mulberry leaf powder, metabolomics, fat deposition, pigs

Received: 19 January 2020

Revised: $\quad 3$ May 2020

Accepted: 15 June 2020

${ }^{3}$ Corresponding author:

e-mail: lifanzhang@njau.edu.cn

\begin{abstract}
As a plant-derived additive, mulberry leaves have been considered a potential feed supplement in livestock production. The present study investigated the metabolic responses in Chinese Erhualian pigs fed a diet with different mulberry leaf powder (MLP) ratios using a metabolomic approach. Three groups of Erhualian pigs were used: $\mathrm{N}$ group (normal chow diet), L group (normal chow diet supplemented with $10 \% \mathrm{MLP}$ ) and $\mathrm{H}$ group (normal chow diet supplemented with 20\% MLP). After 10 weeks, in animals fed 20\% MLP diet reduced average backfat thickness (ABF), leaf fat weight and serum triglyceride (TG) levels were noted, and simultaneously the levels of serum lipase of pigs were increased, while in those fed $10 \%$ MLP diet ABF and serum TG levels were decreased. In comparison to $\mathrm{N}$ group, both $\mathrm{L}$ and $\mathrm{H}$ groups had lower levels of metabolites such as lactic acid, alanine, galactose, phenylalanine and tyrosine, but higher levels of metabolites mainly including malonic and heptadecanoic acids. Pathway analysis revealed that MLP supplementation might alter multiple metabolic processes mainly involved in galactose metabolism and biosynthesis of amino acids. It was indicated that MLP supplementation in pigs reduces fat deposition and alters multiple nutrient metabolism processes, and improves our understanding of the mechanisms involved in metabolic changes induced by MLP diet.
\end{abstract}

\section{Introduction}

Obese pig breeds, including most Chinese native pig breeds, have excellent meat quality, e.g., high intramuscular fat content and moderate muscle marbling (Zhang et al., 1986). However, some of disadvantages in fat traits, such as high fat deposition in subcutaneous and abdominal fat, also exist in these pig breeds. Excessive fat deposition causes poor feed conversion ratio and low lean meat percentage, which greatly increase the costs of feeding and production. Therefore, seeking strategies for reducing fat deposition would contribute to improving the industry of these obese pig breeds.
As one of the plant-derived additives, mulberry leaves have been utilized as green forage in livestock production due to their advantages of high crude protein content and low neutral detergent fibre and production costs (Kandylis et al., 2009; Zhou et al., 2014). Moreover, mulberry leaves or their products such as mulberry leaf powder (MLP) or mulberry leaf extract (MLE) are rich in polyphenolics polysaccharides, and flavonoids (Zhang et al., 2014), which have antioxidant, anti-diabetic or free radical capacity useful in preventing cardiovascular disease, obesity, hyperglycemia and hyperlipidemia of humans or animals (Ann et al., 2015; Trimarco et al., 2015; Zhang et al., 2015; Liu et al., 2016b). 
So, mulberry leaves could be used as a functional food for reducing fat deposition.

So far, the mechanism by which mulberry leaves reduce fat accumulation in humans or animals is still unclear. As an effective and widely used tool to identify key metabolites associated with metabolic responses from pathophysiological and environmental perturbations, metabolome can give us a good opportunity to better understand the biological mechanisms of metabolic changes induced by a diet. Among several metabolomic approaches, gas chromatographymass spectrometry (GC-MS) has excellent sensitivity, high efficiency and straightforward identification of metabolites peaks (Chang and Ho, 2014); and it has been widely used in many metabolomics studies associated with metabolic changes induced by the diet. Additionally, as a typical and famous native pig breed in China, Erhualian pig is a good model for studying the effects of mulberry leaves or their products on fat deposition because this breed has many typical characters, such as high subcutaneous and visceral fat deposition (Liu et al., 2015b).

So, in the present study the metabolic responses caused by the different levels of MLP supplementation in Erhualian pigs using the gas chromatography-quadrupole time-of-flight mass spectrometry (GC-TOF-MS) approach were analysed. The findings provide the comprehensive view on the metabolic changes and their related pathways induced by MLP diet and potential biomarkers associated with lipid-lowering effects in obese pig breeds.

\section{Material and methods}

\section{Ethical approval}

All animal care and handling procedures were approved by the Animal Ethics Committee at Nanjing Agricultural University and Changzhou Science and Technology Bureau (CE20162027).

\section{Animals and diets}

Twenty-one Chinese Erhualian male pigs (6-month old) were raised in the Changzhou Erhualian Pig Production Cooperation (Changzhou, Jiangsu, China). The pigs originated from seven different litters and were housed in individual pens and fed quantitative feeding twice a day, according to Chinese feeding standard of pig (NY/T65-2004). Before starting the experiment, pigs were divided into three groups $(\mathrm{n}=7)$ : control group ( $\mathrm{N}$ group) - pigs fed a normal chow diet, $\mathrm{L}$ group - pigs fed a normal diet supplemented with 10\% MLP as a low dose of MLP, and $\mathrm{H}$ group - pigs fed a normal diet supplemented with $20 \%$ MLP as a high dose of MLP.
MLP was obtained from mulberry leaves of Morus multicaulis, which were harvested from Suqian, Jiangsu (China). After drying, the harvested mulberry leaves were crushed to a uniform size (0.45 mm screen) by a grinder machine (FFC-45K, Qingdao Kangliyongfeng Machinery Manufacturing Co., Ltd, Shandong, China). The contents of conventional nutrients of MLP and feedstuffs were measured according to methods including Kjeldahl determination for crude protein, Soxhlet extraction for crude fat, Chinese national standard GB/T6434-2006 for crude fibre, neutral detergent fibre, and acid detergent fibre, GB/T 6438-2007 for crude ash, GOST 316402012 for dry matter, GB/T6436-2002 for calcium, and GB/T6437-2002 for phosphorus contents.

The MLP diets were fed for 10 weeks. During the trial, the pigs had free access to water and were weighed on day 0 and day 70 .

At the end of the experiment, all pigs were slaughtered in a commercial slaughter house. Blood sample of each animal was obtained using a $500 \mathrm{ml}$ plastic beaker from carotid artery. After $2 \mathrm{~h}$ of condensation in situ, precipitated liquid was collected and centrifuged at $4{ }^{\circ} \mathrm{C}, 3000 \mathrm{~g}$ for $15 \mathrm{~min}$. Serum were collected and stored at $-80{ }^{\circ} \mathrm{C}$. Measurements of backfat thickness (BF) of different body parts on the middorsal line of the left carcass were taken at the shoulder (thickest shoulder, SBF), midback (last rib, MBF) and loin sites (lumbosacral junction, LBF) and then, calculated for average backfat thickness (ABF). The leaf fat (LF) was weighted.

\section{Measuring of serum lipid parameters}

Serum concentrations of total cholesterol (TC), total triglyceride (TG), free fatty acid (FFA), high-density lipoprotein cholesterol (HDL-C) and low-density lipoprotein cholesterol (LDL-C) were measured using an Automatic Chemistry Analyzer (Hitachi 7020, Tokyo, Japan), according the manufacturer's instructions. Serum lipase activities and glucose levels were assayed using commercial kits (A054-2 and F006, respectively) obtained from Nanjing Jiancheng Bioengineering Institute (Nanjing, Jiangsu, China) and analysed by a Microplate Reader (Thermo Fisher Scientific, Waltham, MA, USA).

\section{Sample preparation for GC-TOF-MS}

Serum $(100 \mu \mathrm{l})$ was mixed with $350 \mu$ methanol and $20 \mu \mathrm{L}$-2-chlorophenylalanine (internal standard) and centrifuged at $4{ }^{\circ} \mathrm{C}, 13000 \mathrm{~g}$ for $15 \mathrm{~min}$. The supernatant $(400 \mu \mathrm{l})$ was transferred into a $2 \mathrm{ml}$ GC-MS glass vial. A total of $20 \mu$ solution of each vial was taken and pooled for quality control (QC) 
sample. As suggested by Sangster et al. (2006), five tested serum samples were mixed as a QC sample and processed together with samples. Next, the supernatants in the GC-MS glass vials were dried in a vacuum concentrator without heating. After adding $60 \mu \mathrm{l}$ of methoxyamine hydrochloride $(20 \mathrm{mg} / \mathrm{ml}$ in pyridine) and incubating for $30 \mathrm{~min}$ at $80^{\circ} \mathrm{C}$, each sample was added $80 \mu \mathrm{l}$ of the Bis(trimethylsilyl) trifluoroacetamide (BSTFA) reagent (1\% trimethylchlorosilane, $\mathrm{v} / \mathrm{v}$ ), and then, incubated for $1.5 \mathrm{~h}$ at $70{ }^{\circ} \mathrm{C}$. The QC sample was added a volume of $10 \mu \mathrm{F}$ FAMEs (standard mixture of fatty acid methyl esters, C8-C16: $1 \mathrm{mg} / \mathrm{ml}$; C18-C24: $0.5 \mathrm{mg} / \mathrm{ml}$ in chloroform) after it was cooled to room temperature. Finally, all samples were mixed well for GC-TOF-MS analysis.

\section{GC-TOF-MS analysis}

GC-TOF-MS analysis was described previously (Sun et al., 2015). In brief, an Agilent 7890 gas chromatograph system coupled with a Pegasus HT time-of-flight mass spectrometer (Agilent Technologies, Santa Clara, CA, USA) was applied for GC-TOF-MS analysis. A $1 \mu 1$ aliquot of the sample was injected in splitless mode. Helium was used as the carrier gas. The front inlet purge flow and the gas flow rate through the column were $3 \mathrm{ml} / \mathrm{min}$ and $1 \mathrm{ml} / \mathrm{min}$, respectively. The initial temperature was kept at $50{ }^{\circ} \mathrm{C}$ for $1 \mathrm{~min}$, then raised to $310^{\circ} \mathrm{C}$ by a rate of $20^{\circ} \mathrm{C} / \mathrm{min}$, and then, kept for $6 \mathrm{~min}$ at $310^{\circ} \mathrm{C}$. The injection, transfer line and ion source temperatures were $280^{\circ} \mathrm{C}, 270{ }^{\circ} \mathrm{C}$ and $220^{\circ} \mathrm{C}$, respectively. The energy was $-70 \mathrm{eV}$ in electron impact mode. Data were obtained in full-scan mode with $50-500 \mathrm{~m} / \mathrm{z}$ range at a rate of 20 spectra per second after a solvent delay of $4.7 \mathrm{~min}$.

\section{Data processing}

A Chrome TOF4.3X software and a LECO-Fiehn Rtx 5 database (LECO Corporation, St. Joseph, MI, USA) were used for raw peaks exacting, baselines filtering and calibration, peak alignment, deconvolution analysis, peak identification and integration of the peak area (Kind et al., 2009). The retention time index (RI) method was used in peak identification with 5000 of the RI tolerance. The data quality control was firstly performed by removing metabolites with missing values in more than $30 \%$ of all the individuals. Then, the data were filtered by using the interquartile range method, filling up the missing values of raw data by half of the minimum value and standardizing by the internal standard normalization method.

\section{Metabolite identification and differential metabolite filtering}

Metabolite identification was performed using five databases, including PubChem, Japan Chemical Substance Dictionary Web (NIKKAJI), Human Metabolome Database (HMDB), Chemical Abstracts Service (CAS) and Kyoto Encyclopedia of Genes and Genomes (KEGG). The S-plot from the orthogonal partial least-squares discriminate analysis (OPLS-DA) was used to visualize the metabolites. Differential metabolites were defined as with the similarity values more than 600 , variable importance in projection (VIP) value of 1.0 or more, and $P$-value less than 0.05 after Student's t-test between any two groups.

\section{Pathway analysis}

A MetaboAnalyst was used for pathway construction by employing the human metabolic pathway databases as reference (Xia et al., 2015). Pathways with impact values more than 0.1 were regarded as the relevant pathways, while pathways with $P$-values less than 0.05 and impact values more than 0.1 were defined as the significantly relevant pathways. Pathway analysis of differential metabolites was determined using a KEGG database.

\section{Statistical analysis}

Multivariate statistical analyses, including principal component analysis (PCA), partial least squaresdiscriminant analysis (PLS-DA) and OPLS-DA, were performed using a SIMCA-P 13.0 software (Umetrics, Umea, Sweden). Other statistical analyses were performed using a SPSS v20.0 software (SPSS, Chicago, IL, USA). The differences in body weight, backfat thickness, LF weight and serum lipid parameters were analysed using one-way analysis of variance (ANOVA), followed by Duncan's multiple comparison, and the corrected $P$-values of less than 0.05 or 0.01 were considered significant and highly significant, respectively.

\section{Results}

\section{Diet compositions, body fat content and serum lipid parameters}

In MLP diets, MLP was used to replace a certain proportion of maize and wheat bran in the normal chow diet. So, differences in diet composition among N, L and $\mathrm{H}$ groups were observed. For example, contents of crude fibre, crude ash and calcium in MLP were much higher than those in maize and 
wheat bran (Table 1). Correspondingly, the contents of these ingredients in MLP diet, especially of crude fibre, were higher than that in normal chow (Table 2).

Table 1. Contents of conventional nutrient in mulberry leaf powder (MLP) and feedstuffs, \%

\begin{tabular}{lrccr}
\hline Nutrient levels & MLP & Maize & $\begin{array}{l}\text { Soybean } \\
\text { meal }\end{array}$ & $\begin{array}{l}\text { Wheat } \\
\text { bran }\end{array}$ \\
\hline Dry matter & 89.00 & 86.00 & 89.00 & 87.00 \\
Crude protein & 19.15 & 8.70 & 47.90 & 14.30 \\
Crude fat & 6.61 & 3.60 & 1.50 & 4.00 \\
Crude ash & 14.96 & 1.40 & 4.90 & 4.80 \\
Crude fibre & 19.00 & 1.60 & 3.30 & 6.80 \\
Neutral detergent fibre & 40.66 & 9.30 & 8.80 & 41.30 \\
Acid detergent fibre & 24.21 & 2.70 & 5.30 & 11.90 \\
Calcium & 3.00 & 0.02 & 0.34 & 0.10 \\
Phosphorus & 0.24 & 0.27 & 0.65 & 0.93 \\
\hline
\end{tabular}

Table 2. Composition of the normal chow and mulberry leaf powder (MLP) diets

\begin{tabular}{|c|c|c|c|}
\hline Indices & $\begin{array}{l}\text { Normal } \\
\text { chow diet } \\
\text { (N group) }\end{array}$ & $\begin{array}{l}10 \% \text { MLP } \\
\text { diet } \\
\text { (L group) }\end{array}$ & $\begin{array}{l}20 \% \text { MLP } \\
\text { diet } \\
\text { (H group) }\end{array}$ \\
\hline \multicolumn{4}{|l|}{ Ingredients, \% } \\
\hline maize & 62 & 59 & 56 \\
\hline soybean meal & 20 & 20 & 20 \\
\hline wheat bran & 14 & 7 & 0 \\
\hline mulberry leaf powder & 0 & 10 & 20 \\
\hline premix $^{1}$ & 4 & 4 & 4 \\
\hline \multicolumn{4}{|l|}{ Nutrient levels,$\%$} \\
\hline dry matter & 86.77 & 87.01 & 87.25 \\
\hline digestive energy, MJ /kg & 13.16 & 13.15 & 13.13 \\
\hline crude protein & 16.98 & 17.63 & 18.28 \\
\hline crude fibre & 2.60 & 3.98 & 5.35 \\
\hline ether extract & 3.09 & 3.36 & 3.64 \\
\hline ash & 2.52 & 3.64 & 4.75 \\
\hline calcium & 0.58 & 0.86 & 1.15 \\
\hline phosphorus & 0.50 & 0.46 & 0.41 \\
\hline lysine & 0.82 & 0.82 & 0.83 \\
\hline methionine & 0.26 & 0.26 & 0.26 \\
\hline threonine & 0.61 & 0.62 & 0.64 \\
\hline arginine & 1.05 & 1.05 & 1.04 \\
\hline neutral detergent fibre & 13.31 & 14.20 & 15.10 \\
\hline acid detergent fibre & 4.40 & 5.90 & 7.41 \\
\hline
\end{tabular}

${ }^{1}$ contained per kg of diet: IU: vit. A 11,000, vit. $D_{3} 2,000$, vit. E 20; mg: vit. $K_{3} 1.8$, vit. $B_{1} 2.0$, vit. $B_{2} 5.2$, vit. $B_{6} 3.0$, vit. $B_{12} 0.02$, niacin 30 , pantothenic acid 12 , folic acid 1 , biotin 0.1 , choline chloride 20 , Fe 100, Cu 8, Zn 100, Mn 12, I 0.14, Se 0.1

The changes in body fat content and serum lipid in Erhualian pigs fed MLP diets are shown in Table 3. No significant differences in final body weight were observed between $\mathrm{N}$ and $\mathrm{L}$ or $\mathrm{H}$ groups. The ABF and SBF in L and $\mathrm{H}$ groups were significantly or highly significantly lower than that in $\mathrm{N}$ group
$(P<0.05$ or $P<0.01)$. Among the different parts of body fat deposition, the MBF in L and $\mathrm{H}$ groups were highly significantly lower than that in the $\mathrm{N}$ group $(P<0.01)$, while no significant differences in the LBF were observed between $\mathrm{N}$ and $\mathrm{L}$ or $\mathrm{H}$ groups. In addition, the LF weight in the $\mathrm{L}$ and $\mathrm{H}$ groups showed a significant decrease in comparison to that in the $\mathrm{N}$ group $(P<0.05)$.

It was shown, for serum lipids, that concentrations of $\mathrm{TG}$ in both $\mathrm{L}$ and $\mathrm{H}$ groups were significantly lower than those in the $\mathrm{N}$ group $(P<0.05)$. However, the serum concentrations of TC, HDL-C and LDL-C were similar between N and $\mathrm{L}$ or $\mathrm{H}$ groups. The levels of serum lipase in the $\mathrm{H}$ group were highly significantly higher than those in $\mathrm{N}$ and $\mathrm{L}$ groups $(P<0.01)$. Serum glucose contents in $\mathrm{L}$ and $\mathrm{H}$ groups were significant or highly significantly lower than those in the $\mathrm{N}$ group $(P<0.05$ or $P<0.01$, respectively).

\section{Metabolite detection and identification}

The standard deviation of the retention time of internal standard was 0.001791 , indicating that the GC-TOF-MS analysis was stable. Moreover, a total of 336 peaks were detected and identified using the Chroma TOF4.3X software. Some derivatives were represented by increasing numbers, e.g., serine 1 and serine 2 show derivatives with zero and one trimethylsilyl groups at the primary amino group, respectively (Kind et al., 2009). After quality filtering, 108 metabolites were selected for further analysis.

\section{Comparisons of the metabolite spectra among different groups}

The score plot demonstrated that each sample can be clearly divided, and no outliers were detected (Figure 1). Moreover, in the PLS-DA analysis it was observed that the corresponding R2Y and Q2 values were 0.97 and $0.584,0.993$ and 0.78 , 0.974 and 0.77 between $\mathrm{N}$ and $\mathrm{L}$ groups, $\mathrm{N}$ and $\mathrm{H}$ groups, and $\mathrm{L}$ and $\mathrm{H}$ groups, respectively, while the R2Y and Q2 intercept values of permutation tests were 0.835 and $-0.169,0.94$ and 0.211 , 0.943 and -0.026 between $\mathrm{N}$ and $\mathrm{L}$ groups, $\mathrm{N}$ and $\mathrm{H}$ groups, and $\mathrm{L}$ and $\mathrm{H}$ groups, respectively, indicating that the data have good fitness of model. Besides, the OPLS-DA analysis was performed to maximize the differences between groups in the model. The R2Y and Q2 value of the OPLS-DA analysis were 0.999 and $0.653,0.998$ and $0.7,0.943$ and 0.659 between $\mathrm{N}$ and L groups, $\mathrm{N}$ and $\mathrm{H}$ groups, and $\mathrm{L}$ and $\mathrm{H}$ groups, respectively. Score plots based on OPLS-DA displayed the distinct clustering trends between any two groups (Figure 2A-C). 
Table 3. Body weight, backfat thickness, leaf fat and serum biochemical parameters in Erhualian pigs fed normal chow or mulberry leaf powder (MLP) diet after the 10-week intervention

\begin{tabular}{|c|c|c|c|c|}
\hline Indices & $\mathrm{N}$ group & L group & $\mathrm{H}$ group & $P$-value \\
\hline Initial body weight, $\mathrm{kg}$ & $57.86 \pm 2.29$ & $55.93 \pm 2.10$ & $58.64 \pm 1.61$ & 0.672 \\
\hline Final body weight, kg & $92.29 \pm 3.76$ & $88.93 \pm 3.81$ & $86.57 \pm 1.69$ & 0.522 \\
\hline Average daily feed intake, $\mathrm{kg}$ & $1.58 \pm 0.011$ & $1.59 \pm 0.007$ & $1.59 \pm 0.007$ & 0.734 \\
\hline Average daily gain, $\mathrm{kg}$ & $0.49 \pm 0.03$ & $0.47 \pm 0.03$ & $0.40 \pm 0.01$ & 0.085 \\
\hline Feed conversion ratio & $3.31 \pm 0.18$ & $3.46 \pm 0.22$ & $4.00 \pm 0.13$ & 0.057 \\
\hline $\mathrm{ABF}, \mathrm{mm}$ & $34.04 \pm 1.38^{\mathrm{Aa}}$ & $29.28 \pm 0.71^{\mathrm{ABb}}$ & $27.32 \pm 1.45^{\mathrm{Bb}}$ & 0.006 \\
\hline $\mathrm{SBF}, \mathrm{mm}$ & $43.77 \pm 0.99^{\mathrm{Aa}}$ & $38.82 \pm 1.05^{\mathrm{ABb}}$ & $35.34 \pm 2.14^{\mathrm{Bb}}$ & 0.006 \\
\hline $\mathrm{MBF}, \mathrm{mm}$ & $29.02 \pm 1.54^{\mathrm{A}}$ & $23.02 \pm 1.14^{\mathrm{B}}$ & $22.26 \pm 1.28^{B}$ & 0.007 \\
\hline $\mathrm{LBF}, \mathrm{mm}$ & $29.30 \pm 2.03$ & $25.97 \pm 1.15$ & $24.37 \pm 1.76$ & 0.179 \\
\hline LF weight, $\mathrm{kg}$ & $4.23 \pm 0.30^{\mathrm{a}}$ & $3.37 \pm 0.24^{b}$ & $3.40 \pm 0.11^{b}$ & 0.043 \\
\hline $\mathrm{TG}, \mathrm{mmol} / \mathrm{l}$ & $0.57 \pm 0.02^{\mathrm{a}}$ & $0.49 \pm 0.02^{b}$ & $0.47 \pm 0.02^{b}$ & 0.031 \\
\hline $\mathrm{TC}, \mathrm{mmol} / \mathrm{l}$ & $2.45 \pm 0.14$ & $2.46 \pm 0.09$ & $2.45 \pm 0.15$ & 0.998 \\
\hline $\mathrm{HDL}-\mathrm{C}, \mathrm{mmol} / \mathrm{l}$ & $0.75 \pm 0.04$ & $0.68 \pm 0.04$ & $0.66 \pm 0.04$ & 0.355 \\
\hline LDL-C, mmol/l & $1.51 \pm 0.11$ & $1.57 \pm 0.10$ & $1.59 \pm 0.11$ & 0.893 \\
\hline $\mathrm{FFA}, \mathrm{mmol} / \mathrm{l}$ & $0.37 \pm 0.06$ & $0.48 \pm 0.04$ & $0.60 \pm 0.06$ & 0.052 \\
\hline Activity of lipase, U/l & $7.33 \pm 1.64^{\mathrm{A}}$ & $9.12 \pm 1.11^{\mathrm{A}}$ & $16.07 \pm 1.71^{\mathrm{B}}$ & 0.001 \\
\hline Glucose, $\mathrm{mmol} / \mathrm{l}$ & $7.63 \pm 0.17^{\text {Аа }}$ & $6.81 \pm 0.24^{\mathrm{Ab}}$ & $5.85 \pm 0.19^{B C}$ & 0.000 \\
\hline
\end{tabular}

all data were presented as means \pm standard error, $n=7$ per group; different lowercase and uppercase letters of superscripts in the same row represent significant $(P<0.05)$ and highly significant $(P<0.01)$, respectively; ABF - average backfat thickness of measurements taken at the shoulder, midback and loin sites on the middorsal line; SBF - backfat thickness of the shoulder site; MBF - backfat thickness of the midback site; LBF - backfat thickness of the loin site; LF - leaf fat; FFA - free fatty acid; TG - total triglyceride; TC - total cholesterol; HDL-C - high-density lipoprotein cholesterol; LDL-C - low-density lipoprotein cholesterol. The $\mathrm{N}$ group was fed normal chow diet, while $\mathrm{L}$ and $\mathrm{H}$ groups were fed normal diet supplemented with 10 or $20 \%$ MLP, respectively.

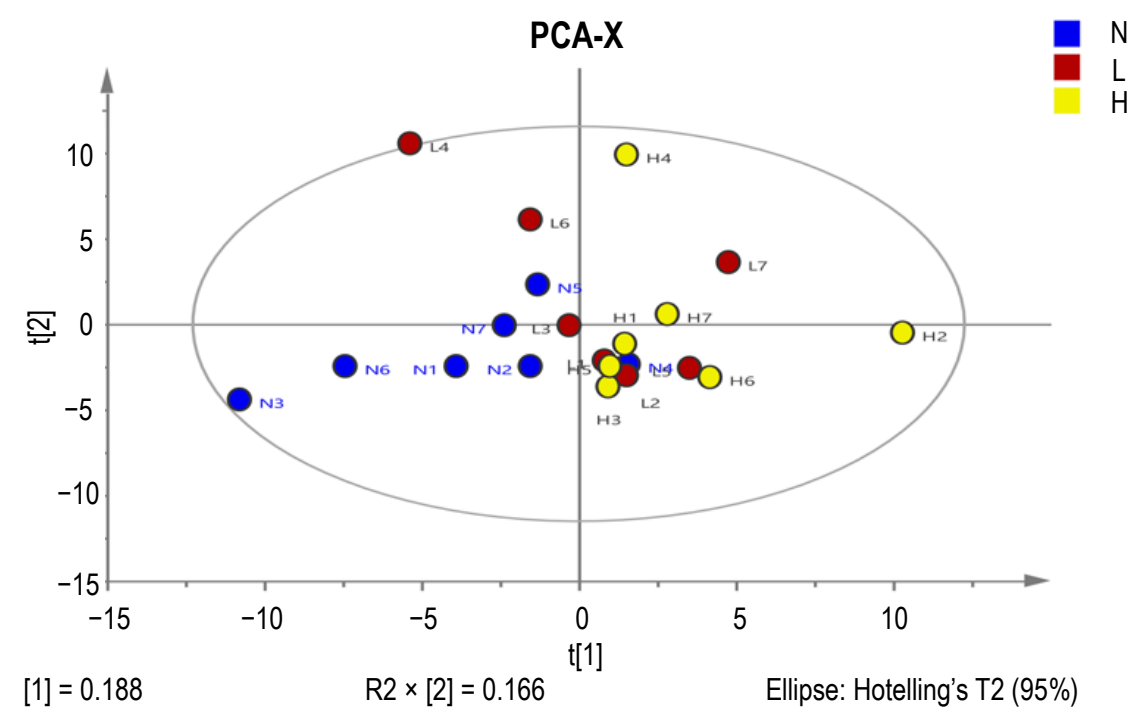

Figure 1. PCA score plot of $N, L$ and $H$ groups. The PCA score plot showed that different samples were approximately scattered into different regions and all samples were in the hostelling T2 ellipse. The X-axis/t[1] and Y-axis/t[2] indicate the first and second principal components, respectively. The $\mathrm{N}$ group was fed normal chow diet, while $\mathrm{L}$ and $\mathrm{H}$ groups were fed normal diet supplemented with 10 or $20 \%$ MLP, respectively.

\section{Identification of differential metabolites among different groups}

Based on criteria of defining differential metabolites, a total of 6,20 and 8 differential metabolites were identified between $\mathrm{N}$ and $\mathrm{L}$ groups, $\mathrm{N}$ and $\mathrm{H}$ groups, and $\mathrm{L}$ and $\mathrm{H}$ groups, respectively (Table 4). In comparison to the $\mathrm{N}$ group, the levels of 14 metabolites in one or two MLP groups sig- nificantly decreased, including lactic acid, glycolic acid, alanine, 3-hydroxybutyric acid, D-glyceric acid, L-malic acid, alpha-ketoglutaric acid, phenylalanine, taurine, ribitol, galactose, tyrosine, myoinositol and indolelactate, while the levels of 4 metabolites in one or two MLP groups significantly increased, including 2-hydroxybutanoic acid, malonic acid, mannose and heptadecanoic acid (Table 4). 


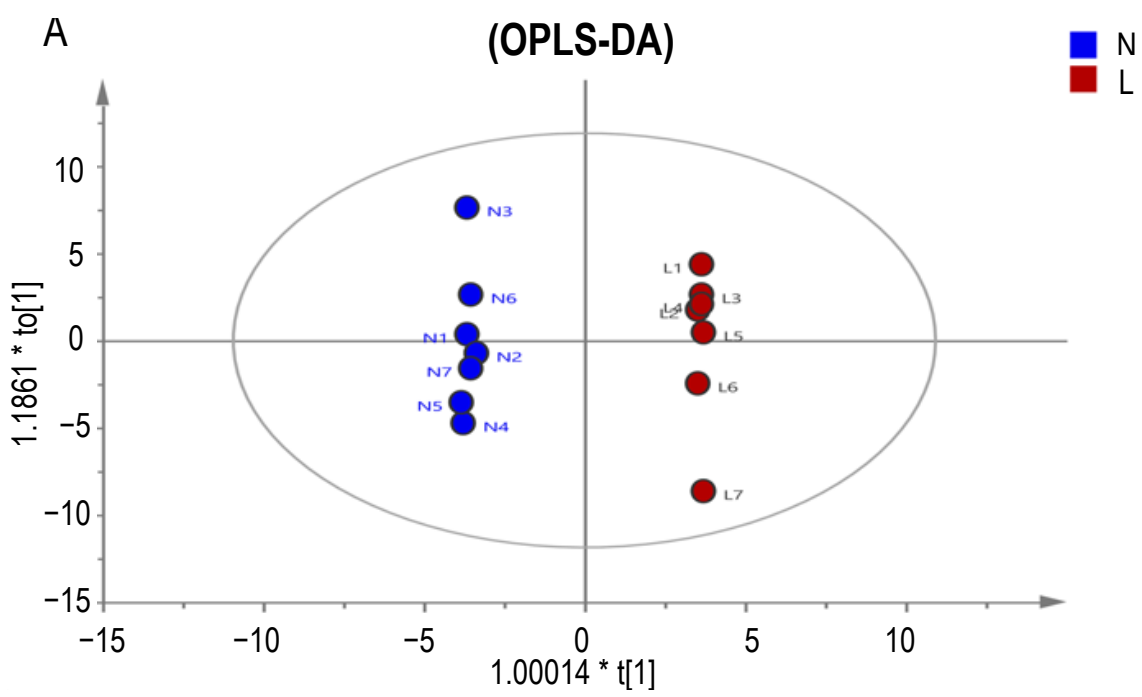

[1] $=0.131 \quad$ R2 $\times$ side Comp. $[1]=0.155 \quad$ Ellipse: Hotelling's T2 $(95 \%)$
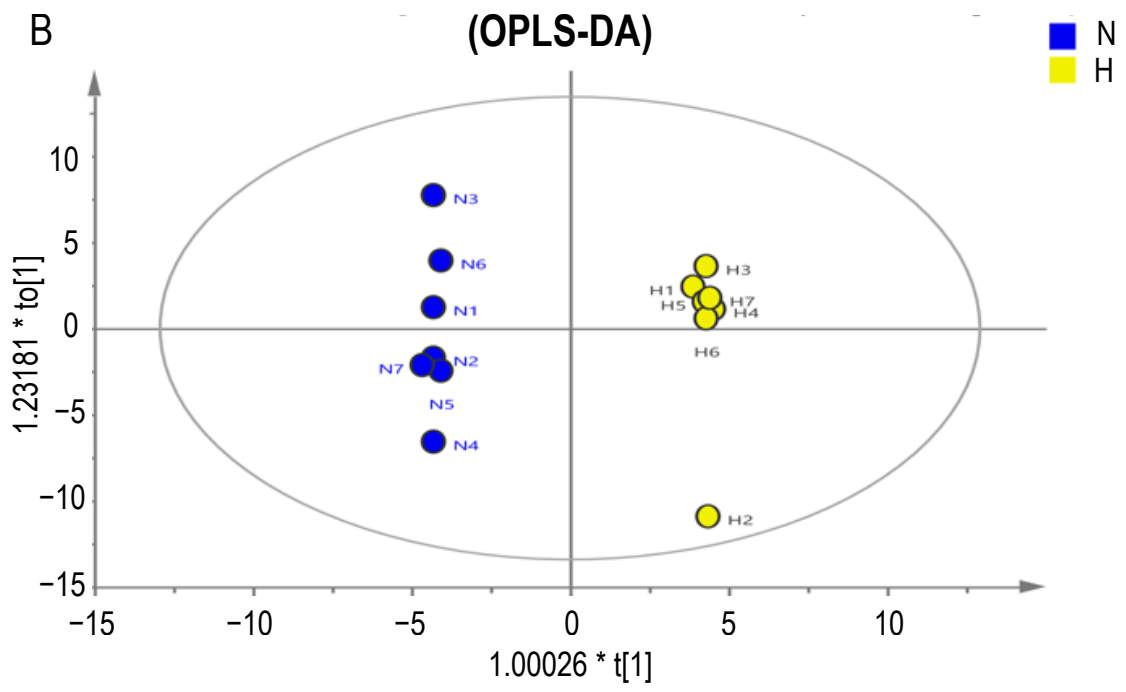

[1] $=0.184 \quad$ R2 $\times[\times$ side Comp. 1] $=0.198 \quad$ Ellipse: Hotelling's T2 (95\%)
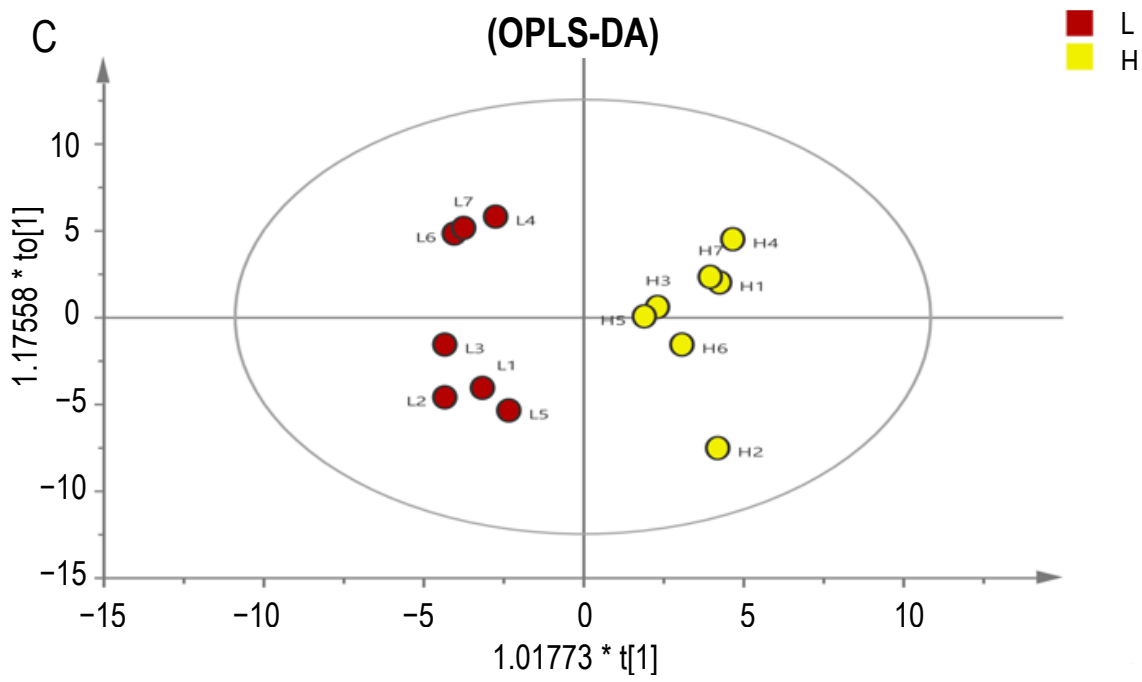

[1] $=0.13 \quad \mathrm{R} 2 \times[\times$ side Comp. 1] $=0.172 \quad$ Ellipse: Hotelling's T2 $(95 \%)$

Figure 2. OPLS-DA analysis of metabolite profiles. The OPLS-DA ( $A, B$ and $C$ ) score plot showed that $N$ and $L$ groups, $N$ and $H$ groups, $L$ and $\mathrm{H}$ groups were scattered into two different regions. The $\mathrm{X}$-axis/t[1] and $\mathrm{Y}$-axis/t[2] indicate the first and second principal components, respectively. The $\mathrm{N}$ group was fed normal chow diet, while L and $\mathrm{H}$ groups were fed normal diet supplemented with 10 or $20 \% \mathrm{MLP}$, respectively. 
Table 4. Identification of differential metabolites among different groups

\begin{tabular}{|c|c|c|c|c|c|c|c|c|c|c|c|c|}
\hline \multirow[b]{2}{*}{ Indices $^{1}$} & \multirow[b]{2}{*}{ Similarity } & \multirow[b]{2}{*}{$\begin{array}{l}\text { Retention } \\
\text { time (RT) }\end{array}$} & \multirow{2}{*}{ Mass } & \multicolumn{3}{|l|}{ N-L } & \multicolumn{3}{|l|}{$\mathrm{N}-\mathrm{H}$} & \multicolumn{3}{|l|}{$\underline{L}-\mathrm{H}$} \\
\hline & & & & $\begin{array}{l}\text { Log2(fold } \\
\text { of change) }\end{array}$ & $P$-value & Vip $p^{3}$ & $\begin{array}{l}\text { Log2(fold } \\
\text { of change) }\end{array}$ & $P$-value & $V_{i p^{3}}$ & $\begin{array}{l}\text { Log2(fold } \\
\text { of change) }{ }^{2}\end{array}$ & $P$-value & Vip ${ }^{3}$ \\
\hline Lactic acid & 933.04 & $5.39494,0$ & 174 & -0.15 & 0.20 & 1.02 & -0.23 & 0.02 & 1.46 & -0.08 & 0.52 & 0.54 \\
\hline Glycolic acid & 893.85 & $5.52534,0$ & 147 & -0.01 & 0.97 & 0.03 & -0.41 & 0.02 & 1.40 & -0.40 & 0.17 & 1.13 \\
\hline Alanine 1 & 967.11 & $5.73435,0$ & 116 & -0.74 & 0.01 & 1.83 & -0.86 & 0.02 & 1.41 & -0.12 & 0.72 & 0.31 \\
\hline 2-hydroxybutanoic acid & 931.04 & $5.89824,0$ & 131 & 0.22 & 0.61 & 0.41 & 1.46 & 0.00 & 1.75 & 1.24 & 0.01 & 2.02 \\
\hline 3-hydroxybutyric acid & 849.96 & $6.15506,0$ & 147 & -0.35 & 0.13 & 1.17 & -0.59 & 0.02 & 1.40 & -0.23 & 0.34 & 0.81 \\
\hline N-methyl-DL-alanine & 840.11 & $6.25096,0$ & 130 & -0.17 & 0.65 & 0.37 & 1.44 & 0.02 & 1.44 & 1.61 & 0.01 & 1.88 \\
\hline Malonic acid 1 & 617.78 & $6.49216,0$ & 147 & 1.31 & 0.00 & 2.11 & 1.04 & 0.01 & 1.61 & -0.27 & 0.11 & 1.30 \\
\hline D-glyceric acid & 892.00 & $7.3633,0$ & 73 & -0.50 & 0.08 & 1.32 & -0.55 & 0.00 & 1.72 & -0.06 & 0.86 & 0.15 \\
\hline L-malic acid & 895.74 & $8.41682,0$ & 73 & -0.55 & 0.13 & 1.18 & -0.57 & 0.04 & 1.31 & -0.02 & 0.96 & 0.04 \\
\hline Threitol & 907.26 & $8.51833,0$ & 217 & 0.35 & 0.13 & 1.17 & -0.40 & 0.13 & 0.98 & -0.75 & 0.02 & 1.73 \\
\hline Oxoproline & 945.11 & $8.7038,0$ & 156 & 0.42 & 0.09 & 1.31 & -0.10 & 0.75 & 0.22 & -0.52 & 0.00 & 2.19 \\
\hline Alpha-ketoglutaric acid & 898.26 & $8.9734,0$ & 198 & -0.02 & 0.95 & 0.05 & -0.67 & 0.02 & 1.45 & -0.65 & 0.18 & 1.10 \\
\hline Glutamic acid & 841.11 & $9.24028,0$ & 246 & 0.02 & 0.92 & 0.08 & -0.71 & 0.04 & 1.31 & -0.73 & 0.02 & 1.73 \\
\hline Phenylalanine 1 & 918.11 & $9.34136,0$ & 218 & -0.14 & 0.29 & 0.84 & -0.68 & 0.02 & 1.40 & -0.54 & 0.05 & 1.53 \\
\hline Xylose 1 & 786.71 & $9.43625,0$ & 103 & -0.19 & 0.77 & 0.23 & 1.07 & 0.04 & 1.28 & 1.26 & 0.03 & 1.65 \\
\hline Taurine & 884.07 & $9.5808,0$ & 326 & -0.34 & 0.18 & 1.05 & -1.06 & 0.01 & 1.57 & -0.72 & 0.05 & 1.56 \\
\hline Ribitol & 812.11 & $9.78719,0$ & 217 & -0.12 & 0.69 & 0.32 & -0.70 & 0.04 & 1.27 & -0.59 & 0.13 & 1.23 \\
\hline D-(glycerol 1-phosphate) & 766.22 & $10.0278,0$ & 357 & 0.61 & 0.18 & 1.04 & -0.41 & 0.36 & 0.62 & -1.02 & 0.04 & 1.61 \\
\hline Mannose 1 & 846.74 & $10.7012,0$ & 274 & 1.17 & 0.03 & 1.57 & 0.78 & 0.09 & 1.09 & -0.39 & 0.42 & 0.68 \\
\hline Galactose 1 & 840.15 & $10.8023,0$ & 103 & -0.50 & 0.05 & 1.49 & -0.29 & 0.04 & 1.27 & 0.22 & 0.40 & 0.70 \\
\hline Tyrosine 1 & 916.78 & $11.0257,0$ & 218 & -0.69 & 0.02 & 1.72 & -0.73 & 0.02 & 1.42 & -0.04 & 0.87 & 0.14 \\
\hline Myo-inositol & 942.74 & $11.7416,0$ & 217 & -0.29 & 0.13 & 1.17 & -0.43 & 0.04 & 1.28 & -0.14 & 0.62 & 0.42 \\
\hline Heptadecanoic acid & 601.76 & $12.0118,0$ & 117 & 1.08 & 0.00 & 1.96 & 1.22 & 0.01 & 1.53 & 0.14 & 0.59 & 0.46 \\
\hline Indolelactate 2 & 737.19 & $12.1617,0$ & 202 & -0.49 & 0.18 & 1.04 & -0.89 & 0.03 & 1.33 & -0.40 & 0.42 & 0.68 \\
\hline
\end{tabular}

${ }_{1}^{1}$ derivatives were named by increasing numbers according to retention index from the FiehnLib library, e.g., serine 1, serine 2 and serine 3 show the derivatives with zero, one or two trimethylsilyl groups at the primary amino group, respectively; ${ }^{2}$ fold change of group was calculated as the latter group dividing the former group using mean of normalized intensity of each metabolite; ${ }^{3}$ Vip - value of variable importance in projection (VIP); '+' sign refers to abundance increase in latter group, while '-' sign refers to abundance decrease in latter group; the N groups were fed normal chow diet, while $\mathrm{L}$ and $\mathrm{H}$ groups were fed normal diet supplemented with 10 or $20 \%$ MLP, respectively.
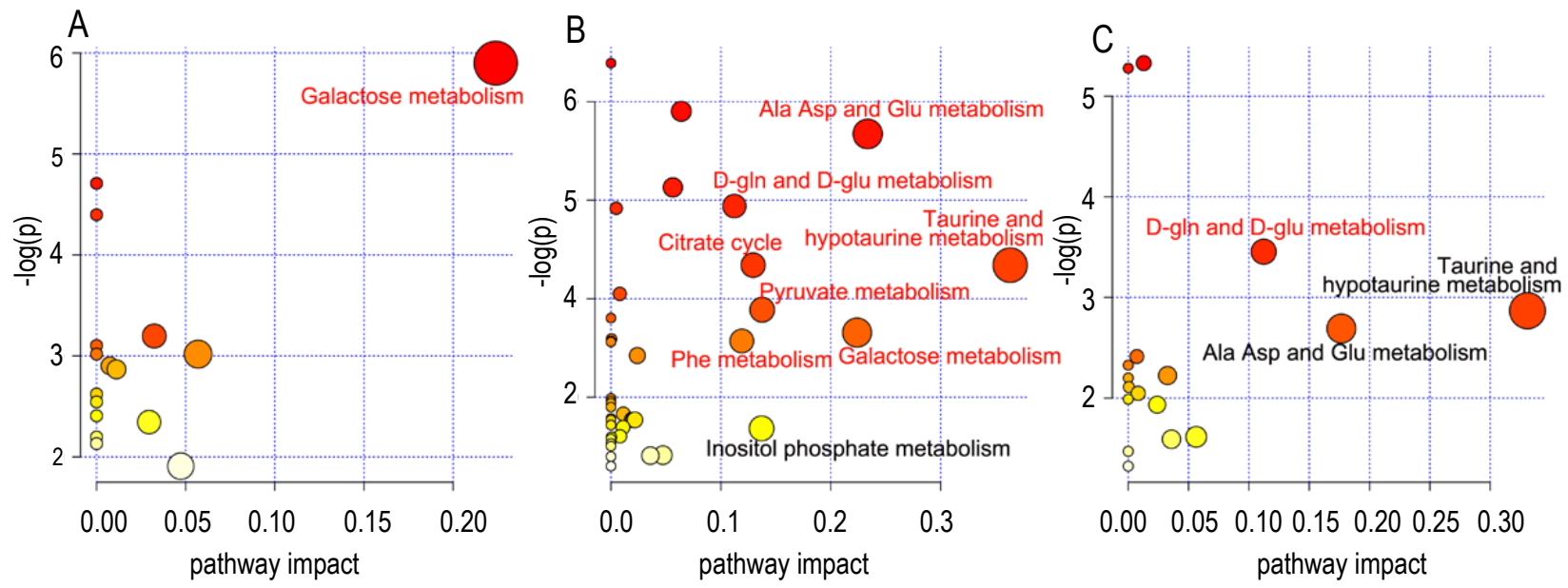

Figure 3. Pathway view of the differential metabolites among $N, L$ and $H$ groups. (A) Relevant pathways identified between $N$ and $L$ groups; (B) Relevant pathways identified between $\mathrm{N}$ and $\mathrm{H}$ groups; (C) Relevant pathways identified between $\mathrm{L}$ and $\mathrm{H}$ groups. The $\mathrm{x}$-axis represents pathway impact, while the $y$-axis represents pathway enrichment. The power of pathway enrichment was showed by the size and colour of pathways, while the significantly relevant pathways were marked with red text annotation on the diagram. The $\mathrm{N}$ groups were fed normal chow diet, while $L$ and $H$ groups was fed normal diet supplemented with 10 or $20 \%$ MLP, respectively. 


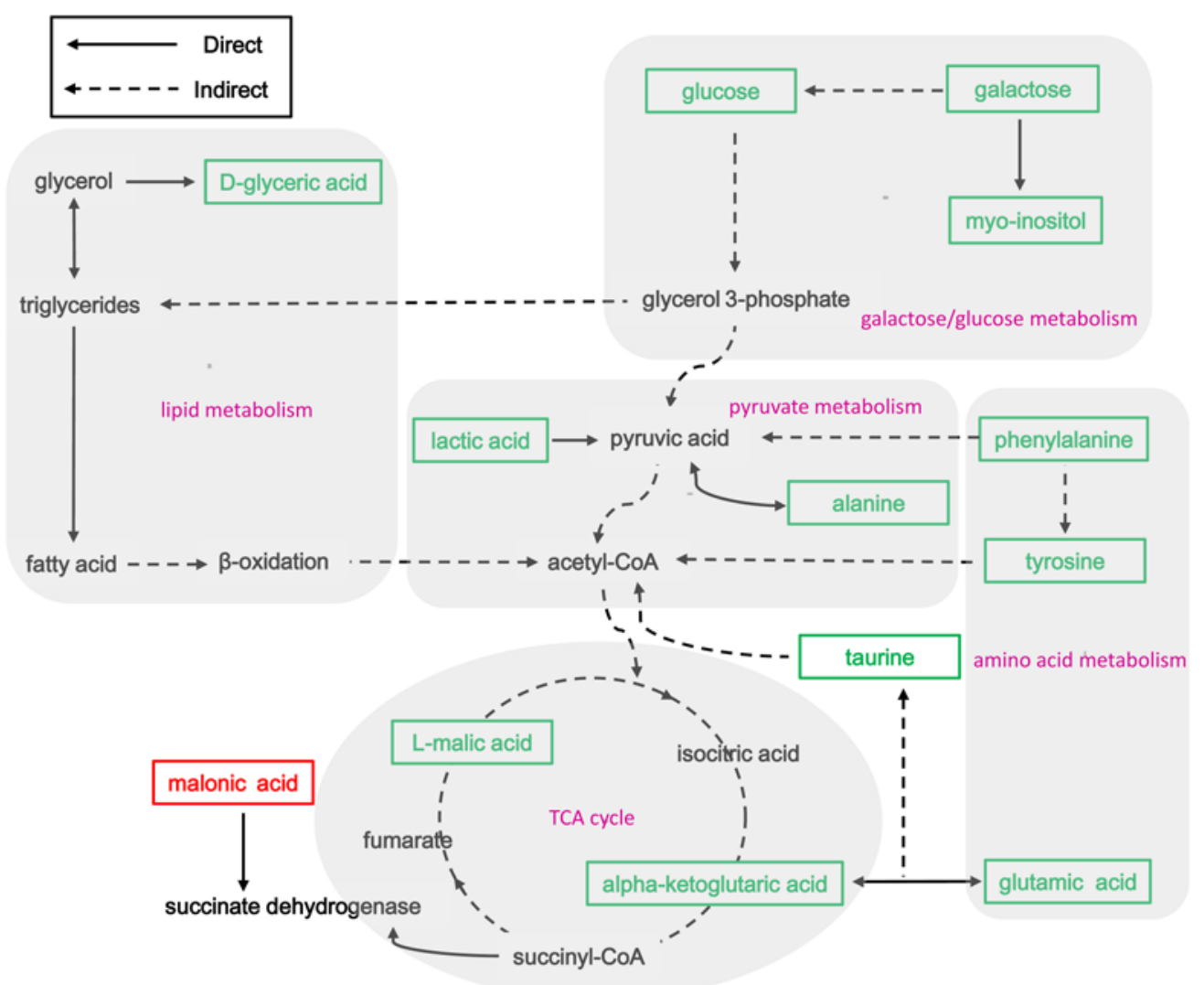

Figure 4. KEGG pathways associated with differential metabolites. KEGG pathways associated with differential metabolites are manually linked together. The illustration was generated using the reference maps from KEGG to construct the altered pathways of biosynthesis of amino acids, pyruvate metabolism, galactose metabolism, taurine and hypotaurine metabolism, TCA cycle, alanine, aspartate and glutamate metabolism, phenylalanine metabolism and beta-alanine metabolism. Colourful words with border denote the differential metabolites discovered in this study. Compared to $\mathrm{N}$ group, metabolites marked in red colour represent significantly up-regulated metabolites in one or two MLP groups, while metabolites marked in green colour represent significantly down-regulated metabolites in one or two MLP groups. The N groups were fed normal chow diet, while $L$ and $H$ groups were fed normal diet supplemented with 10 or $20 \%$ MLP, respectively.

\section{Metabolic pathway and network analysis}

To explore the difference of metabolic mechanism caused by MLP, metabolic pathways and their related network were built further. A total of 1, 8 and 3 relevant pathways were found between $\mathrm{N}$ and $\mathrm{L}$ groups, $\mathrm{N}$ and $\mathrm{H}$ groups, and $\mathrm{L}$ and $\mathrm{H}$ groups (Figure $3 \mathrm{~A}-\mathrm{C}$ ), respectively. Of these, seven pathways including: galactose metabolism, citrate cycle (TCA cycle), alanine, aspartate and glutamate metabolism, D-glutamine and D-glutamate metabolism, taurine and hypotaurine metabolism, phenylalanine metabolism and pyruvate metabolism, were characterized as significantly relevant pathways (Figure $3 \mathrm{~A}-\mathrm{C}$ ). Furthermore, the KEGG network analysis of 12 differential metabolites from the above significantly relevant pathways showed that galactose metabolism, biosynthesis of amino acids, pyruvate metabolism, taurine and hypotaurine metabolism, TCA cycle, alanine, aspartate and glutamate metabolism, phenylalanine metabolism and beta-alanine metabolism were the main pathways associated with lipid and energy metabolism (Figure 4).

\section{Discussion}

In the present study, MLP is used to investigate the lipid-lowering effect for improving the production efficiency of Erhualian pigs. It is well known that $\mathrm{BF}$ and LF are two key phenotypic indicators of body fat deposition in pigs. In recent studies it was found that mulberry leaves and MLP diet can significantly reduce single-point $\mathrm{BF}$ and TG levels of crossbred pigs and ABF of Chinese Xiangcun Black pigs, respectively (Liu et al., 2019; Fan et al., 2020). Similar to these observations, we have also indicated that MLP diet significantly decreased ABF, SBF, MBF, LF and serum TG levels, with no significant influence on body weight of Erhualian pigs. In previous studies it was observed that mulberry leaves or MLE reduce serum TG concentrations and enhance subcutaneous fat lipolysis in crossbred pigs or liver lipolysis in high-fat diet-induced obese mice (Ann et al., 2015; Peng et al., 2018; Fan et al., 2020). Consistently, we had provided a new evidence that MLP can reduce body fat deposition in pigs. 
Moreover, serum levels of glucose in $\mathrm{L}$ and $\mathrm{H}$ groups were found to be significantly lower than those in the $\mathrm{N}$ group. In previous studies it was reported that 1-deoxynojirimycin (DNJ, one of mulberry extracts) and mulberry feeding could decrease levels of glucose in mice or rats (Liu et al., 2015a; Liu et al., 2016a). We have shown that MLP diet decreased glucose levels in pigs. Additionally, the levels of other carbohydrates, such as galactose and myo-inositol, were also found to be significantly decreased in $\mathrm{L}$ or $\mathrm{H}$ groups in comparison to those in the N group. These results imply that MLP supplementation might decrease galactose/glucose metabolism. We have observed that the levels of D-glyceric acid were lower in groups with MLP and significantly decreased in the $\mathrm{H}$ group. D-glyceric acid is a direct product of glycerol, while glycerol and glycerol 3-phosphate are materials for synthesized TG. Besides, glycerol 3-phosphate is synthesized by dihydroxyacetone phosphate, an intermediate product of glycolysis. Therefore, reducing levels of glucose and D-glyceric acid might indirectly decrease the levels of TG via regulating glycerol 3-phosphate and glycerol, respectively. However, more studies are needed to investigate this speculation. Another compound influencing the glycolysis is lactic acid, which content in our study was lower in groups fed diet with MLP addition. Lactic acid is an end product of glycolysis and can directly regulate pyruvic acid, a main product of glycolysis pathway for regulating energy metabolism (Shi et al., 2015). The content of alanine, another metabolite synthesized by pyruvic acid, was also lower in animals fed diets supplemented with MLP. These data was in accordance with a previous report, in which MLP diet reduced levels of plasma glucose, pyruvic acid and lactic acid in diabetic rats (Andallu and Varadacharyulu, 2007). To our knowledge, pyruvic acid can convert into acetyl-CoA, which is a key factor for lipid metabolism and TCA cycle. Reduction of lactic acid and alanine might result in decreased pyruvic acid contents, thus impacting on lipid metabolism via acetyl-CoA. Consistently with the previous research showing that MLP or DNJ by decreasing glucose or pyruvate metabolic pathways can have antiobesity effects (Andallu and Varadacharyulu, 2007; $\mathrm{Hu}$ et al., 2017), it can be suggested from our data that MLP might affect lipid and energy metabolisms via regulating the pathways associated with galactose/glucose and pyruvate metabolisms.

Furthermore, MLP supplementation induced metabolic alterations related to energy metabolism by regulating TCA cycle. Some potential biomarkers associated with TCA cycle, such as malonic acid, L-malic acid and alpha-ketoglutaric acid, were identified in this study. Malonic acid is a well-characterized competitive inhibitor of succinate dehydrogenase (Quastel and Wooldridge, 1928), indicating that malonic acid can directly regulate TCA cycle. The levels of two differential metabolites involved in TCA cycle in the $\mathrm{H}$ group, including L-malic acid and alpha-ketoglutaric acid, were significantly reduced in comparison to those in the $\mathrm{N}$ group, implying that energy production of TCA cycle might slow down in pigs fed diets with high content of MLP. The role of mulberry leaves in energy metabolism has not been fully investigated yet. In the most recent study using liquid chromatography-mass spectrometry (LC-MS) analysis it was found that DNJ might increase the levels of TCA intermediates in DNJ-treated diabetic mice (Hu et al., 2017). We have noted that the effect of MLP on TCA cycle is ambiguous and further investigations are needed to recognise the role of MLP in regulating TCA cycle.

As we have expected, we have shown that MLP diet had an impact on metabolic alterations associated with amino acid metabolism. MLP diets decreased the levels of several important amino acids including phenylalanine, tyrosine and taurine. Phenylalanine is an essential amino acid and has been shown to influence lipid metabolism (Pohle-Krauza et al., 2008; Ueda et al., 2016). Previously, DNJ was found to promote the conversion of phenylalanine to acetyl-CoA (Liu et al., 2015a; Hu et al., 2017). Tyrosine, a hydroxylation product of phenylalanine (Bequette et al., 1999), is a semi-essential amino acid depending on dietary intake (Matthews, 2007). During tyrosine catabolism process, the carboxyl carbon is released and the other part of tyrosine transfers into acetoacetate or fumarate (Sun et al., 2016), which can be utilized to synthesize fatty acids and demonstrated that tyrosine could have an impact on the synthesis of fatty acids. Therefore, reduction of tyrosine in pigs fed MLP diets might be associated with the decrease of lipogenesis. Taurine is a potentially essential amino acid and its supplementation reduced serum TG and prevented high-fat diet-induced obesity with increased energy expenditure in rats or mice (Nishimura et al., 2002; Tsuboyama-Kasaoka et al., 2006), which is not fully consistent with our data. Actually, the role of taurine in lipid metabolism is still controversial. For example, taurine supplementation was shown to promote lipid synthesis in fish (Shen et al., 2018). The exact role of taurine in lipid and energy metabolism induced by MLP needs further investigation. Our data provide a comprehensive clue on the action of MLP in amino acid metabolism. 
Additionally, supplementation of MLP changed the levels of other well-known metabolites related to lipid metabolism, e.g., heptadecanoic acid and 3-hydroxybutyric acid. As an odd-chain fatty acid and a biomarker of fat intake, heptadecanoic acid has been found to be inversely associated with the human adiposity or type 2 diabetes (Forouhi et al., 2014; Aglago et al., 2017), showing that heptadecanoic acid can negatively regulate fat metabolism. In the present study, significantly increased heptadecanoic acid in MLP groups, accompanied by the decrease of lipid indicators, strongly supports this speculation. 3-hydroxybutyric acid, known as beta-hydroxybutyrate, is a ketone body and produced from acetylCoA derived from $\beta$-oxidation of fatty acids (Laffel, 1999). Previously, in many studies it was found that beta-hydroxybutyrate can reduce or inhibit adipocyte lipolysis in cows and mice (Taggart et al., 2005; van der Drift et al., 2013). We have shown that MLP supplementation reduced the levels of 3-hydroxybutyric acid and body fat deposition, indicating that MLP supplementation could enhance lipolysis by decreasing the levels of 3-hydroxybutyric acid.

\section{Conclusions}

For the first time, the changes in metabolic profiles during mulberry leaf powder (MLP) consumption in Erhualian pigs have been investigated in this study. MLP diet regulated multiple lipid homeostasisrelated metabolites and their pathways to reduce body fat deposition in pigs. A basis for further investigations of the role of these potential MLP-related biomarkers was not only established, but also a novel clue of understanding the mechanism of the body fat changes induced by MLP supplementation was provided.

\section{Acknowledgments}

This work was supported by the Natural Science Foundation of Jiangsu Province of China (BK20181319) and the National Key Technology Research and Development Program of the Ministry of Science and Technology of China (2015BAD03B01).

\section{References}

Aglago E.K., Biessy C., Torres-Mejia G., Angeles-Llerenas A., Gunter M.J., Romieu I., Chajes V., 2017. Association between serum phospholipid fatty acid levels and adiposity in Mexican women. J. Lipid. Res. 58, 1462-1470, https://doi. org/10.1194/jr.P073643

Andallu B., Varadacharyulu N.C., 2007. Gluconeogenic substrates and hepatic gluconeogenic enzymes in streptozotocin-diabetic rats: effect of mulberry (Morus indica L.) leaves. J Med Food 10, 41-48, https://doi.org/10.1089/jmf.2005.034
Ann J.Y., Eo H., Lim Y., 2015. Mulberry leaves (Morus alba L.) ameliorate obesity-induced hepatic lipogenesis, fibrosis, and oxidative stress in high-fat diet-fed mice. Genes. Nutr. 10, 46, https://doi.org/10.1007/s12263-015-0495-x

Bequette B.J., Backwell F.R., Kyle C.E., Calder A.G., Buchan V., Crompton L.A., France J., MaCrae J.C., 1999. Vascular sources of phenylalanine, tyrosine, lysine, and methionine for casein synthesis in lactating goats. J. Dairy. Sci. 82, 362-377, https://doi.org/10.3168/jds.S0022-0302(99)75243-4

Chang K.L., Ho P.C., 2014. Gas chromatography time-of-flight mass spectrometry (GC-TOF-MS)-based metabolomics for comparison of caffeinated and decaffeinated coffee and its implications for Alzheimer's disease. PLoS ONE 9, e104621, https://doi.org/10.1371/journal.pone.0104621

Fan L., Peng Y., Wu D., Hu J., Shi X., Yang G., Li X., 2020. Dietary supplementation of Morus nigra $L$. leaves decrease fat mass partially through elevating leptin-stimulated lipolysis in pig model. J. Ethnopharmacol. 249, 112416, https://doi. org/10.1016/j.jep.2019.112416

Forouhi N.G., Koulman A., Sharp S.J. et al., 2014. Differences in the prospective association between individual plasma phospholipid saturated fatty acids and incident type 2 diabetes: the EPIC-InterAct case-cohort study. Lancet Diabetes. Endocrinol. 2, 810-818, https://doi.org/10.1016/S22138587(14)70146-9

Hu X.Q., Thakur K., Chen G.H., Hu F., Zhang J.G., 2017. Metabolic effect of 1-deoxynojirimycin from mulberry leaves on $\mathrm{db} /$ $d b$ diabetic mice using liquid chromatography-mass spectrometry based metabolomics. J. Agric. Food Chem. 65, 4658-4667, https://doi.org/10.1021/acs.jafc.7b01766

Kandylis K., Hadjigeorgiou I., Harizanis P., 2009. The nutritive value of mulberry leaves (Morus alba) as a feed supplement for sheep. Trop. Anim. Health. Prod. 41, 17-24, https://doi. org/10.1007/s11250-008-9149-y

Kind T., Wohlgemuth G., Lee D.Y., Lu Y., Palazoglu M., Shahbaz S., Fiehn O., 2009. FiehnLib: mass spectral and retention index libraries for metabolomics based on quadrupole and time-offlight gas chromatography/mass spectrometry. Anal. Chem. 81, 10038-10048, https://doi.org/10.1021/ac9019522

Laffel L., 1999. Ketone bodies: a review of physiology, pathophysiology and application of monitoring to diabetes. Diabetes. Metab. Res. Rev. 15, 412-426, https://doi.org/10.1002/(SICI)15207560(199911/12)15:6<412::AID-DMRR72>3.0.CO;2-8

Liu Q., Li X., Li C., Zheng Y., Peng G., 2015a. 1-Deoxynojirimycin alleviates insulin resistance via activation of insulin signaling PI3K/AKT pathway in skeletal muscle of $d b / d b$ mice. Molecules 20, 21700-21714, https://doi.org/10.3390/molecules201219794

Liu Q., Li X., Li C., Zheng Y., Wang F., Li H., Peng G., 2016a. 1-deoxynojirimycin alleviates liver injury and improves hepatic glucose metabolism in $\mathrm{db} / \mathrm{db}$ mice. Molecules 21, 279, https:// doi.org/10.3390/molecules21030279

Liu X., Xiong X., Yang J. et al., 2015b. Genome-wide association analyses for meat quality traits in Chinese Erhualian pigs and a Western Duroc x (Landrace x Yorkshire) commercial population. Genet. Sel. Evol. 47, 44, https://doi.org/10.1186/ s12711-015-0120-x

Liu Y., Li X., Xie C., Luo X., Bao Y., Wu B., Hu Y., Zhong Z., Liu C., Li M., 2016b. Prevention effects and possible molecular mechanism of mulberry leaf extract and its formulation on rats with insulin-insensitivity. PloS ONE 11, e0152728, https:// doi.org/10.1371/journal.pone. 0152728 
Liu Y., Li Y., Peng Y., He J., Xiao D., Chen C., Li F., Huang R., Yin Y., 2019. Dietary mulberry leaf powder affects growth performance, carcass traits and meat quality in finishing pigs. J. Anim. Physiol. Anim. Nutr. (Berl) 103, 1934-1945, https:// doi.org/10.1111/jpn.13203

Matthews D.E., 2007. An overview of phenylalanine and tyrosine kinetics in humans. J. Nutr. 137, 1549S-1555S; discussion 1573S-1575S, https://doi.org/10.1093/jn/137.6.1549S

Nishimura N., Umeda C., Ona H., Yokogoshi H., 2002. The effect of taurine on plasma cholesterol concentration in genetic type 2 diabetic GK rats. J. Nutr. Sci. Vitaminol. 48, 483-490, https:// doi.org/10.3177/jnsv.48.483

Peng C.H., Lin H.T., Chung D.J., Huang C.N., Wang C.J., 2018. Mulberry leaf extracts prevent obesity-induced NAFLD with regulating adipocytokines, inflammation and oxidative stress. J. Food Drug Anal. 26, 778-787, https://doi.org/10.1016/j. jfda.2017.10.008

Pohle-Krauza R.J., Navia J.L., Madore E.Y., Nyrop J.E., Pelkman C.L., 2008. Effects of L-phenylalanine on energy intake in overweight and obese women: interactions with dietary restraint status. Appetite 51, 111-119, https://doi.org/10.1016/j.appet.2008.01.002

Quastel J.H., Wooldridge W.R., 1928. Some properties of the dehydrogenating enzymes of bacteria. Biochem. J. 22, 689-702, https://doi.org/10.1042/bj0220689

Sangster T., Major H., Plumb R., Wilson A.J., Wilson I.D., 2006. A pragmatic and readily implemented quality control strategy for HPLC-MS and GC-MS-based metabonomic analysis. The Analyst. 131, 1075-1078, https://doi.org/10.1039/b604498k

Shen G., Huang Y., Dong J., Wang X., Cheng K.K., Feng J., Xu J., Ye J., 2018. Metabolic effect of dietary taurine supplementation on Nile Tilapia (Oreochromis nilotictus) evaluated by NMRbased metabolomics. J. Agric. Food. Chem. 66, 368-377, https://doi.org/10.1021/acs.jafc.7b03182

Shi Z., Yu H., Sun Y., Yang C., Lian H., Cai P., 2015. The energy metabolism in caenorhabditis elegans under the extremely lowfrequency electromagnetic field exposure. Sci. Rep. 5, 8471, https://doi.org/10.1038/srep08471

Sun H., Wang B., Wang J., Liu H., Liu J., 2016. Biomarker and pathway analyses of urine metabolomics in dairy cows when corn stover replaces alfalfa hay. J. Anim. Sci. Biotechnol. 7, 49, https://doi.org/10.1186/s40104-016-0107-7

Sun H.Z., Wang D.M., Wang B., Wang J.K., Liu H.Y., Guan le L., Liu J.X., 2015. Metabolomics of four biofluids from dairy cows: potential biomarkers for milk production and quality. J. Proteome. Res. 14, 1287-1298, https://doi.org/10.1186/s40104016-0107-7
Taggart A.K., Kero J., Gan X. et al., 2005. (D)-beta-Hydroxybutyrate inhibits adipocyte lipolysis via the nicotinic acid receptor PUMA-G. J. Biol. Chem. 280, 26649-26652, https://doi. org/10.1074/jbc.C500213200

Trimarco V., Izzo R., Stabile E., Rozza F., Santoro M., Manzi M.V., Serino F., Schiattarella G.G., Esposito G., Trimarco B., 2015. Effects of a new combination of nutraceuticals with Morus alba on lipid profile, insulin sensitivity and endotelial function in dyslipidemic subjects. A cross-over, randomized, doubleblind trial. High Blood Press. Cardiovasc. Prev. 22, 149-154, https://doi.org/10.1007/s40292-015-0087-2

Tsuboyama-Kasaoka N., Shozawa C., Sano K., Kamei Y., Kasaoka S., Hosokawa Y., Ezaki O., 2006. Taurine (2-aminoethanesulfonic acid) deficiency creates a vicious circle promoting obesity. Endocrinology 147, 3276-3284, https://doi.org/10.1210/ en.2005-1007

Ueda K., Nakamura Y., Yamaguchi M., Mori T., Uchida M., Fujita S., 2016. Amino acid mixture enriched with arginine, alanine, and phenylalanine stimulates fat metabolism during exercise. Int. J. Sport. Nutr. Exerc. Metab. 26, 46-54, https://doi. org/10.1123/ijsnem.2015-0137

van der Drift S.G., Everts R.R., Houweling M., van Leengoed L.A., Stegeman J.A., Tielens A.G., Jorritsma R., 2013. Effects of beta-hydroxybutyrate and isoproterenol on lipolysis in isolated adipocytes from periparturient dairy cows and cows with clinical ketosis. Res. Vet. Sci. 94, 433-439, https://doi. org/10.1016/j.rvsc.2012.11.009

Xia J., Sinelnikov I.V., Han B., Wishart D.S., 2015. MetaboAnalyst 3.0--making metabolomics more meaningful. Nucleic Acids Res. 43, W251-W257, https://doi.org/10.1093/nar/gkv380

Zhang Q., Lu Y., Ma Z., Li Y., Guo J., Meng Q., Bian H., 2015. A novel formula from mulberry leaf ameliorates diabetic nephropathy in rats via inhibiting the TGF-beta1 pathway. Food Funct. 6, 3307-3315, https://doi.org/10.1039/C5FO00711A

Zhang Y., Ren C., Lu G., Cui W., Mu Z., Gao H., Wang Y., 2014. Purification, characterization and anti-diabetic activity of a polysaccharide from mulberry leaf. Regul. Toxicol. Pharmacol. 70, 687-695, https://doi.org/10.1016/j.yrtph.2014.10.006

Zhang Z.G., Li B.D., Chen X.H., 1986. Pig Breeds in China. Shanghai Scientific and Technical Publisher. Shanghai (China)

Zhou Z., Zhou B., Ren L., Meng Q., 2014. Effect of ensiled mulberry leaves and sun-dried mulberry fruit pomace on finishing steer growth performance, blood biochemical parameters, and carcass characteristics. PLoS ONE 9, e85406, https://doi. org/10.1371/journal.pone.0085406 